\title{
28 Research Square \\ Comparative analysis of the complete chloroplast genome of seven Nymphaea species
}

chunqing Sun

Zhen jiang institute of agricultural science in Jiangsu hilly areas

fadi Chen

Nanjing Agricultural University

nianjun Teng

Nanjing Agricultural University

yuemei Yao

Zhenjiang institute of agricultural science in Jiangsu hilly areas

\section{Xi Shan}

Zhenjiang institute of agricultural science in Jiangsu hilly areas

zhongliang Dai ( $\sim$ daizhongliang2008@126.com)

Zhenjiang institute of agricultural of the science of the Jiang-zhen hilly district

\section{Research article}

Keywords: water lily, chloroplast genome, phylogenetic analysis

Posted Date: March 31st, 2020

DOl: https://doi.org/10.21203/rs.3.rs-20050/v1

License: (c) (i) This work is licensed under a Creative Commons Attribution 4.0 International License.

Read Full License

Version of Record: A version of this preprint was published at Aquatic Botany on March 1st, 2021. See the published version at https://doi.org/10.1016/j.aquabot.2021.103353. 


\section{Abstract}

Background: Although there has been a long history of cultivation and research on Nymphaea, the taxonomic relationships and evolutionary relationships among Nymphaea species remain controversial. The chloroplast (cp) genome can provide a new method to determine species origin, evolution, and phylogenetic relationships of Nymphaea. However, there are few studies on the cp genomes of Nymphaea, and the data of their genomes are very scarce. The complete cp genomes of seven Nymphaea species were sequenced by high-throughput sequencing technology, and the structural characteristics and phylogenetic relationships of $\mathrm{cp}$ genomes were analyzed.

Results: A total of 126-129 genes were annotated in seven Nymphaea species, including 81-84 protein coding genes, eight rRNA, and 37 tRNA genes. A comparative cp genomic analysis of seven Nymphaea species showed that the cp gene sequence of Nymphaea was consistent, with no signs of reverse rearrangement. The codons preferentially ended with A/U. The cp genomes of seven Nymphaea species contained 147-168 dispersed repeats with a length of 15-19 bp and 979 simple sequence repeats (SSRs). Using $N$. colorata as the reference sequence, a total of 8,328 single nucleotide polymorphisms (SNPs) and 1,579 insertions/deletions were obtained. The degree of variation of the cp genome of the seven Nymphaea species in $r p o A-r p / 20, r b c L-n d h C, n d h D-n d h F$, and $t r n N-G U U-n d h A$ regions is relatively high; related regions can be used as potential molecular markers for population genetics research. KaKs analysis showed that the ycf2 gene was positively selected. The results of the phylogenetic analysis showed that the genus Nymphaea can be divided into five branches: subgenus Nymphaea, subgenus Hydrocallis, subgenus Lotos, subgenus Anecphya, and subgenus Brachyceras.

Conclusions: The cp genome of Nymphaea is very conservative in structure and composition, but it has rich variation in LSC and SSC regions. The phylogenetic analysis showed that Nymphaea could be further divided into five subgenera, and Euryale and Victoria were most closely related to Nymphaea. This study provided insight on the structure and composition of the cp genome of Nymphaea. Moreover, our results have provided more sequence information and genome resources for follow-up studies on Nymphaea.

\section{Background}

Nymphaea, one of the important aquatic flowers, is located at the root of the angiosperm tree [1, 2]. Nymphaea consists of approximately 50 species and is distributed on all continents except Antarctica [3, 4]. Nymphaea is divided into five subgenera based up on the morphology of flowers, seeds, leaves, and stems, and the geographical distribution, namely, subgenus Nymphaea, subgenus Hydrocallis, subgenus Lotos, subgenus Anecphya, and subgenus Brachyceras [5]. Subgenus Anecphya contains six or seven species only distributed in Australia and New Guinea. There are 10-12 species in subgenus Brachyceras, which are widely distributed in the tropics and subtropics. Subgenus Hydrocallis is composed of 14 species that are distributed in the neotropics, primarily in South America. Subgenus Lotos contains one to three species that are distributed in the tropics of the Old World. Subgenus Nymphaea contains six to seven species that are distributed in temperate areas of the Northern Hemisphere. However, there are still 
some controversies on the phylogenetic classification of Nymphaea. For example, based on the noncoding cp markers, some studies have supported the division of Nymphaea genera into three subgenera『Subgenus Nymphaea followed by Subgenus Anecphya-Brachyceras and another clade comprising Subgenus Hydrocallis-Lotos [6, 7]. In addition, relationships among species within Nymphaea remain unclear. For example, using the $\mathrm{cp} t r n T-\operatorname{trnF}$ sequences to construct phylogenetic trees of Nymphaeaceae, a previous study has supported three subbranches within Subgenus Nymphaea [7]. However, a phylogeny was constructed using the internal transcribed spacers (ITS) region of nrDNA and the study supported the division of Subgenus Nymphaea into two subbranches [8]. There are different understandings of the evolutionary relationships among Nymphaea species; therefore, it is of great significance to study its phylogenetic relationship.

The cp genome is relatively conservative in gene composition and structure compared with the nuclear and mitochondrial genomes $[9,10]$. Because of its small size and relatively conservative structure, the $\mathrm{cp}$ genome has become an ideal model for evolutionary and comparative genomic research [11], and its structure and sequence information are of great value in revealing the origin, evolution, and relationships among different species. With the rapid development of high-throughput sequencing technology, research on the cp genome has increased $[12,13]$. However, there are few studies on the $\mathrm{cp}$ genomes of Nymphaea, and the data of their genomes are very scarce.

In this study, the cp genomes of seven species of Nymphaea were sequenced by high-throughput sequencing technology. The full length of $\mathrm{cp}$ genomes was obtained and the composition, structure, and phylogenetic relationships of $\mathrm{cp}$ genomes were analyzed in order to enrich the genetic information of Nymphaea and provide a theoretical basis for future research on phylogeography, phylogenetics, and conservation of Nymphaea.

\section{Results}

\section{The basic structural characteristics of the cp genome of seven species of Nymphaea}

After high-throughput sequencing, raw data was obtained. Low quality reads were removed, leaving the remaining paired-end reads of seven Nymphaea species. The paired-end reads ranged from 19,642,319 reads for $N$. odorata to $26,112,273$ reads for $N$. micrantha. After performing de novo genome sequencing and assembly, seven complete cp genome sequences for $N$. odorata, $N$. rubra, N. gigantea, $N$.

potamophila, $N$. colorata, $N$. tetragona, and $N$. micrantha were obtained (Fig 1; Table 1 ) and submitted to GenBank under the following accession numbers: MT107636, MT107632, MT107637, MT107633, MT107631, MT107634, and MT107635, respectively.

These seven novel $\mathrm{cp}$ genome sequences had the classical quadripartite structure that contained one LSC, one SSC, and two IR (IRa and IRb) regions (Fig 1). The cp genomes of seven species of Nymphaea had different sizes, among which the largest was $N$. gigantea at 160,179 bp long and the smallest was $N$. potamophila at 159,232 bp (Table 1). The length of the LSC ranged from 89,450-90,266 bp, SSC ranged from 19,340-1,965 bp, and IR ranged from 25,163-25,232 bp (Table 1). Therefore, the variation of the 
length of the LSC is greater than that of SSC and IR, and the difference in genome length was mainly due to the varying lengths of the LSC and SSC. The GC content of the seven Nymphaea cp genomes was $\sim 39 \%$ (Table 1); the GC contents in IR ( 43\%) of these seven Nymphaea species were higher than that of LSC and SSC regions ( 37\% and 34\%).

A total of 126-129 genes were identified in the cp genome of seven Nymphaea species, with 126, 127, and 128 in N. odorata, N. rubra, and N. tetragona, respectively, and 129 in the other four species (Table 1). Most of the genes are single-copy genes, but the six protein coding genes (rps12, rp/23, rp/2, ycf2, rps7, and $n d h B$ ), seven tRNA genes (trnl-CAU, trnL-CAA, trnV-GAC, trnl-GAU, trnA-UGC, trnR-ACG, and trnN-GUU) and four rRNA genes (rrn16s, rrn23s, rrn4.5s, and rrn5s) in the IR region contain two copies (Table 2). In the cp genome of the seven Nymphaea species, the number of rRNAs and tRNAs was the most conservative, with eight and 37, respectively (Table 1). There were 81-84 protein coding genes (Table 1; Table 2), among which ycf1 was missing in $N$. tetragona, ycf 4 and atpF were missing in $N$. rubra. $n d h F$, rpoc2, and $y c f 1$ were missing in $N$. odorata.

The IR and SC boundaries of the cp genomes of the seven Nymphaea species were compared and analyzed. As shown in Figure 2, the LSC/IRb boundaries of the seven Nymphaea species were all within the $r p / 2$ gene, and the length of the overlapping region between the boundary and the rpl2 gene was 15$39 \mathrm{bp}$. The IRb/SSC boundaries of the six species were between $\operatorname{trnN}$ and $n d h F$, and the length of the $n d h F$ gene entering SSC was 49-80 bp. However, the SSC region of $N$. odorata lacked an $n d h F$ gene and there was a 522 bp gap between the boundary and the $t r n N$ gene in the IRb region. The SSC/IRa boundaries of five species were all located in ycf1gene, the length of the gene in the SSC region was between 5,659-5,719 bp, and was between 155-167 bp in the IRa region. No ycf1 gene was found in the SSC regions of $N$. odorata or $N$. tetragona, and there were 521 bp and 545 bp gaps between the trnN gene in the border and IRa regions, respectively.

\section{Codon preference}

The codon preferences of the cp genome of seven Nymphaea species were analyzed, and there was no significant difference among the genomes. $N$. colorata was used as an example to determine the codon preference of the cp genome of Nymphaea. A total of 25,995 codons were detected in 79 protein coding genes of the $N$. colorata cp genome and their RSCU values were calculated (Additional file 1). Among these codons, 2,636 were used to encode leucine (Leu), accounting for $10.1 \%$ of all codons, with UUA being the most commonly used. The content of codons used to code Tryptophan (Trp) was the lowest, 447 in total, accounting for $1.7 \%$ of all codons. The RSCU values of 32 codons were greater than 1 , of which 28 ended with $A / U$, indicating that the codon ending preference was $A / U$. The RSCU of tryptophan (Trp) was equal to 1 , indicating that Trp has no codon preference.

\section{Analysis of dispersed repeats and simple repeat sequences (SSRs)}

The dispersed repeats of the cp genome include forward, reverse, palindrome, and complementary types. Using Vmatch v2.3.0 software, the dispersed repeat sequences of seven Nymphaea species were 
identified. For N. odorata, N. rubra, N. gigantea, N. potamophila, N. colorata, N. micrantha, and N. tetragona, $163,147,151,158,153,155$, and 168 repeat sequences, respectively, with a length of $15-19$ bp were detected in the $\mathrm{cp}$ genome and were distributed in the coding region, the gene interval region, and the gene intron region (Table 3). There was little difference in the total number of dispersed repeats of seven Nymphaea species, and the length of the sequences was concentrated between 15-19 bp, accounting for $83.4-88.0 \%$ of the total dispersed repeats (Table 3 ).

A total of 979 SSRs had been identified in the cp genome of seven Nymphaea species (Table 3). Among these SSRs, the most abundant was trinucleotide SSR, accounting for $48.2 \%$ (472), followed by mononucleotide SSR $(44.7 \%$; 438), dinucleotide SSR $(3.3 \%$; 32$)$, tetranucleotide SSR $(2.9 \% ; 28)$, pentanucleotide SSR $(1.1 \% ; 11)$, and hexanucleotide SSR $(0.1 \% ; 1)$ (Table 3). Pentanucleotide SSRs were distributed in N. gigantea (AAGAA, ATTTA, TATAT, and TTATA), N. micrantha (CATAA), N. odorata (ATACC), N. rubra (AAAGG and TTCCT), and N. tetragona (ATACC, TATTA, and TTAGC); a hexanucleotide SSR was only distributed in N. gigantea (ATTTAT) (Table 3). Most of the SSRs of the seven species were located in the LSC (56.1-59.7\%), followed by the SSC (20.3-21.6\%) and the IR (18.8-21.9\%).

\section{Collinearity, KaKs, Pi, SNP, and indel analysis}

Although the cp genome of plants is conservative, there will be rearrangement over the course of evolution. In this study, we selected the $\mathrm{cp}$ genome of $N$. colorata as the reference sequence for comparison with the other six cp genomes of Nymphaea. The results showed that there was no reverse rearrangement in the $\mathrm{cp}$ genomes of the seven species of Nymphaea, indicating a collinear relationship (Fig 3). In addition, the sequence differences of $105-115 \mathrm{~kb}$ of the $\mathrm{cp}$ genome of the seven species of Nymphaea are large (Fig 3); therefore, it can be used to develop molecular markers for future genetic analyses of Nymphaea.

The synonymous substitution rate (Ka), missense substitution rate (Ks), and $\mathrm{Ka} / \mathrm{Ks}$ of seven species of Nymphaea were calculated by using the $\mathrm{cp}$ genome of $N$. colorata as the reference sequence (Additional file 2). Most of the cp genes have low Ka and Ks, indicating the conservation of the evolutionary process of the $\mathrm{cp}$ genome. $\mathrm{Ka} / \mathrm{Ks}<1$ of most genes indicate that they may undergoing purifying selection, i.e., the mutation is harmful and eliminated in the population. Among the genomes, the Ka/KS of $y c f 2$ in the six comparison groups, $N$. colorata vs. $N$. gigantea, $N$. colorata vs. N. micrantha, N. colorata vs. $N$. odorata, $N$. colorata vs. $N$. potamophila, $N$. colorata vs. $N$. rubra, and $N$. colorata vs. $N$. tetragona were all greater than 1 , indicating that $y c f 2$ was subject to positive selection effect.

The Pi values of eight genes (petN, petG, $p s a\lfloor p s b F\rfloor p s b L \square s b M \square p s b N \rrbracket a n d r p s 18$ ) in the LSC region, all

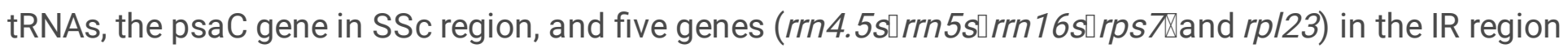
were 0 (Additional file 3), indicating that these genes were relatively conserved in the seven Nymphaea species. Some genes had relatively high Pi values, for example, rp/32, rps19, rp/20, and $y c f 1$ had Pi values of $0.012579,0.011947,0.009195$, and 0.006307 , respectively, indicating that the greater the number of polymorphisms in these genes in Nymphaea, the more abundant their genetic diversity. These regions of 
$r p / 32-n d h F, r p s 19-p s b N$, and $a t p B-n d h J$ in the cp genome of Nymphaea were highly variable, indicating that they may be potential molecular markers (Fig 4).

With $N$. colorata as the reference sequence, 8,328 SNPs and 1,579 insertions/deletions (indels) were obtained by global genome alignment using MAFFT software (Additional file 4; Additional file 5). SNPs were found in both coding and non-coding regions of $\mathrm{cp}$ genome. However, there was a relatively large number of SNPs in some highly variable regions located in $r p o A-r p / 20, r b c L-n d h C, n d h D-n d h F$, and

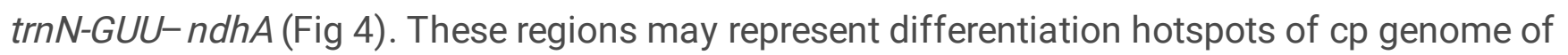
Nymphaea. Moreover, $64.7 \%$ of SNPs $(5,391)$ were located in the intergenic region; the remaining SNPs were located in $84 \mathrm{cp}$ genes (Additional file 4). In addition, some genes, such as matK, $n d h A, n d h F, r p o C 1$, rрoc2, rpoA, rpoB, $a c c D$, atpA, $y c f 1$, and $y c f 2$, all showed a high density of SNPs (Fig 4; Additional file 4). We obtained 1,579 indels mutations, $93.3 \%$ of which were located in the gene interval and only $6.7 \%$ (106) of them were located in the protein coding genes (Additional file 5). These 106 indels were distributed in matK, rps11, rp/2, rp/22, rp/32, rpoC2, petB, ndhF, ndhJ, accD, atpE, ycf1, ycf2, and ycf4 genes.

\section{Phylogenetic analysis}

Nymphaeaceae was divided into five main branches, Brasenia, Nelumbo, Nuphar, Nymphaea, and Cabomba, but Euryale and Victoria were closely related to Nymphaea (Fig 5). Nymphaea can be further divided into five subbranches, corresponding to the five Nymphaea subgenera (Fig 5). The first branch consists of $N$. gigantea from the subgenus Anecphya; the second subfamily consists of $N$. colorata, $N$. micrantha, $N$. capensis, and $N$. ampla which compose subgenus Brachyceras. The third branch is the subgenus Nymphaea, which is composed of $N$. mexicana, $N$. odorata, $N$. alba var. rubra, $N$. alba, and $N$. tetragona; N. potamophila and N. jamesoniana compose the fourth branch, subgenus Hydrocallis. The fifth branch is the subgenus Lotos, which contains of $N$. lotus and $N$. rubra.

\section{Discussion}

\section{The structure and composition of cp genome of Nymphaea}

The cp genome of Nymphaea is composed of LSC, SSC, IRa, and IRb regions, and the overall length of the $\mathrm{cp}$ genome within middle and upper ranges of angiosperms (159-160 kb) [14]. Although the structure and composition of the cp genome of seven Nymphaea are very similar, a comparative analysis shows that there are also many variations in the microstructure. The SSC and LSC regions contain the most variation, while multiple copies of the two IR regions make the IR region less prone to mutation [15]. In this study, $N$. gigantea and the other six species had a large difference in cp genome length, while $N$. odorata and $N$. tetragona, as well as $N$. colorata and $N$. micrantha had similar cp genome length, which revealed that $N$. gigantea was distant from the other six species, while $N$. odorata and $N$. tetragona, $N$. colorata and $N$. micrantha were closely related. The difference in the cp genome size was mainly caused by the contraction and expansion of the IR [14]. However, the difference of the cp genome size of Nymphaea was primarily related to the length variation of LSC and SSC. Gene loss in the cp genome was 
common, for example, gene ycf1 and ycf2 were completely lost in Gramineae [16], and gene infA was lost in many angiosperm cp genomes $[17,18]$. In this study, the cp genome of seven Nymphaea species also had gene loss and the $y c f 1$ gene disappeared completely in $N$. odorata and $N$. tetragona. It is unknown whether $y c f 1$ was transferred to the nuclear or mitochondrial genome. The GC content of the $\mathrm{cp}$ genomes of seven Nymphaea species showed differences in four regions, with the highest content in the IR region (43.35-43.44\%), while the GC content in the LSC and SSC regions was lower, which is due to the existence of rRNA and tRNA in IR region. When the GC content in genome is greater, it has a greater the density of DNA bases, making the DNA sequence more stable and difficult to mutate [14].

The IR region is the most conservative region in the $\mathrm{cp}$ genome, but it also shrinks and expands during evolution [19]. Gene trnH-GUG and ndhF often appeared at the boundary of IR and SC region of cp gene, which were considered to be footprints of $\mathrm{cp}$ genome evolution [20]. We analyzed the contraction and expansion of IR region and found that the gene distribution at the boundary of the four regions of the $\mathrm{cp}$ genome had the same rule, but differences were observed in the microstructure, especially the locations of rps19, ndhF, ycf1, and trnH-GUG. According to the different locations of these four genes, $N$. tetragona and $\mathrm{N}$. odorata can be identified from the other Nymphaea species. The boundary of SSC and IRa region is located in gene ycf1. The expansion of the IR region into ycf1 had also been described in Cardiocrinum [21] and Amana [22]. Therefore, rps19, ndhF, ycf1, and trnH-GUG can be considered part of the evolutionary footprint of the cp genome of Nymphaea.

\section{Codon preference}

In this study, 67 codons were used in seven Nymphaea species, with 32 preferred codons (RSCU>1). The most commonly used codon in the cp genome is the codon with a higher AU content [23], and the preferred codon tends to end with A/U [24]. In this study, only UUG and AUG codons of the 32 preferred codons were G-terminal and the other preferred codons were A/U-terminal. Leucine (Leu) was the most encoded amino acid, and its synonymous codon preference was $U U A>U U G>C U U>C U A>C U G>C U C$, which is inconsistent previous studies conducted in other families $[16,25]$. The codon usage is related to gene expression level, base composition, asymmetric mutation of DNA strands, and selection [26], but the mechanism of codon usage requires further study. The results of this study provide insight into the evolution of the cp genome of Nymphaea.

\section{Repeat sequence analysis}

In this study, the length of the genome repeat sequence of seven Nymphaea species was between 15-19 bp and no large segment repeat sequence (>100 bp) reported in other species was found [27, 28]. Large fragment repeats are related to gene rearrangement [29]. For example, Erodium, Pelargonium, and Geranium (Geraniaceae) have gene rearrangements that all contain large fragment repeats (>100 bp) [9]. The results of the collinearity analysis of seven Nymphaea species showed that all the genes are collinear and no rearrangement occurs, which is consistent with the result that there is no large segment repeat sequence in cp genome. 
SSR markers are highly polymorphic and are therefore used as molecular markers in population genetics and studies of evolution. Most SSRs in the cp genomes of seven Nymphaea species are trinucleotide SSRs (48.2\%), which is inconsistent with the main SSR types of other plants [30, 31]. For example, most SSRs in the cp genomes of Primula are single nucleotide SSRs [25]. In addition, in the gene coding region, the number of SSRs in ycf1 is the largest, which is consistent with the research conducted in Primula [25] and Cardiocrinum [21]. The SSRs in the cp genome reported in this study can be used as potential molecular markers in future studies on Nymphaea.

\section{Nucleotide polymorphism, KaKs, SNP and indel analysis}

The Pi values of the LSC and SSC regions were significantly higher than that of the IR region, which was related to the conservation of the IR region. Three gene spacer regions, $r p / 32-n d h F, r p s 19-p s b N$, and atp $B-n d h J$, as well as four protein coding regions, $r p / 32, r p s 19, r p / 20$, and $y c f 1$, have been reported in other plants as highly variable regions, and they are also considered potential molecular markers [27, 3233]. These regions may undergo faster nucleotide substitution rates during the course of evolution, which is of great significance to phylogenetic analysis and identification of Nymphaea.

Our result showed that $y c f 2$ in the cp genome of Nymphaea was under positive selection. ycf2 was located in the reverse repeat region of $\mathrm{cp}$ genome, which is an important enzyme coding gene in the $\mathrm{cp}$ [34]. The evolutionary rate of $y c f 2$ is relatively fast, its gene function is controversial, and it has not been classified into a main functional gene type (genetic system gene and photosynthetic system gene) [35]. However, because of its cp ATPase coding function [34], it is believed to have a regulatory role in the development of fruit and flower organs [35], which is considered one of the important genes in the cp genome. Sites undergoing positive selection were detected in gymnosperms and other angiosperms, indicating that the evolution of $y c f 2$ is widespread in plants [36]. The seven Nymphaea species in this study are distributed on different continents and grow under quite different environmental conditions. Therefore, the positive selection of ycf2 in Nymphaea may be related to the difference of their optimal growth environment and the different demand for light intensity. The discovery of positive selection on

ycf 2 confirmed that there was evolution at the molecular level in the process of environmental adaptation for Nymphaea.

Large indels are uncommon among the $\mathrm{cp}$ genomes of the same genus [15]. Similarly, for the cp genome of Nymphaea, most of indels exist in the noncoding region, and the largest indel is only $28 \mathrm{bp}$ long. Compared with the small number of indels, we found 8,328 SNPs in the cp genome of Nymphaea. These SNPs and indel regions have a rapid rate of evolution and the degree of variation was the greatest in the $r p o A-r p / 20, r b c L-n d h C, n d h D-n d h F$, and $t r n N-G U U-n d h A$ intergenic regions. These data indicate that these regions may be suitable DNA barcodes for Nymphaea.

\section{Phylogenetic analysis}

In this study, a phylogenetic tree were constructed with 21 species of seven genera in Nymphaeaceae. Cabomba was found to be at the base of the phylogenetic tree. Euryale, Victoria, and Nymphaea 
subgenus Hydrocallis and subgenus Lotos formed a clade, indicating that the three genera are closely related. Using $r b c L$, matK, and $18 S$ rRNA sequences to construct phylogenetic trees of Nymphaeaceae, a previous study has supported the establishment of Cabombaceae by separating Cabomba and Brasenia from Nymphaeaceae [37]. However, Cabomba and Brasenia are not sister to each other in this study, indicating that it is not appropriate to combine them to form Cabombaceae. At present, most researchers agree that Nymphaeaceae is composed of eight genera, Nuphar, Barclaya, Ondinea, Nymphaea, Euryale, Brasenia, Victoria, and Cabomba [37-39]. Our results are consistent with the previous classification of Nymphaeaceae.

Phylogenetic trees were constructed for 15 Nymphaea species and the results showed that Nymphaea can be divided into five subgenera, which is consistent with the previous classification [8]. However, the relationship between species in the genus Nymphaea is still unclear and needs further study. In this study, subgenus Anecphya, subgenus Hydrocallis, and subgenus Lotos form separate branches indicating support for these subgenera, but subgenus Nymphaea and subgenus Brachyceras showed further divisions. Subgenus Brachyceras can be divided into two branches, one containing $N$. capensis and $N$. colorata, the other composed of $N$. ampla and $N$. micrantha. Subgenus Nymphaea can also be divided into two branches, one containing $N$. odorata and $N$. mexicana, and the other containing $N$. alba and $N$. tetragona. The division of subgenus Nymphaea and subgenus Brachyceras may be caused by different growing environments. Our results are consistent with the results presented by Dkhar et al. [8]. The genetic relationship between subgenus Nymphaea and subgenus Brachyceras, subgenus Brachyceras and subgenus Anecphya, subgenus Hydrocallis and subgenus Lotos is relatively close, which is also supported by their cross compatibility [40-42].

\section{Conclusions}

The complete cp genomes of seven Nymphaea species were sequenced and successfully constructed. The cp genome of Nymphaea is very conservative in structure and composition, but it has rich variation in LSC and SSC regions, which can be used for germplasm identification, phylogenetic analysis, and evolution research. The phylogenetic analysis showed that Nymphaea could be further divided into five subgenera, and Euryale and Victoria were most closely related to Nymphaea. This study not only enriched the genetic information on Nymphaea, but also laid a theoretical foundation for the evaluation of water lily germplasm resources, molecular breeding, development of SSR molecular markers, and research into genetic diversity. Moreover, this study provided support for the resolution of the phylogenetic relations among Nymphaea species.

\section{Methods}

\section{Plant materials}

Seven Nymphaea species, N. odorata, N. rubra, N. gigantea, N. potamophila $\mathbb{N}$. tetragona, N. colorata, and $N$. micrantha, were included in this study. Herbarium specimens are all preserved at the Zhenjiang 
Agricultural Science and Technology Innovation Center and live plants were introduced to the Shanghai Chenshan Botanical Garden. These plants were identified by Nianjun Teng and Yingchun Xu of Nanjing Agricultural University according to their key morphological characteristics provided in Huang et al. [5], and the voucher ID were listed in Table 1. In August 2019, fresh leaves were collected, wrapped with tinfoil, frozen in liquid nitrogen, and stored at $-80^{\circ} \mathrm{C}$.

\section{Extraction and sequencing of whole genome DNA}

Whole genome DNA was extracted with the plant genomic DNA Extraction Kit (TIANGEN Beijing China). After testing the genomic DNA of the sample, DNA was fragmented by sonication, and then purified by fragment purification, terminal repair, 3 '-terminal plus A, linked sequencing adapter, and agarose gel electrophoresis for fragment size selection, and PCR amplified into a sequencing library. After passing quality inspection, the constructed library was sequenced with an Illumina Novaseq platform. After sequencing, raw data was filtered out to remove the joint sequence and low-quality reads to obtain highquality clean data.

\section{Cp genome assembly}

First, we used Bowtie 2 V2.2.4 (http://bowtie-bio.sourceforge.net/bowtie2/index.shtml) software to compare the $\mathrm{cp}$ genome database built by Nanjing Jisi Huiyuan Biotechnology Co., Ltd. and selected the matching clean reads for subsequent assembly. We then assembled the filtered clean reads with SPAdes V3.10.1 (http://cab.spbu.ru/software/spades/) software. If the cyclic gene sequence could be directly obtained, the sequence was genome corrected, and then the corrected genome was rearranged to obtain the complete cyclic cp genome sequence. If the complete circular genome was not directly obtained, we used SSPACEV2.0 (http://www.baseclear.com/services/bioinformatics/basetools/sspace-standard/) software to connect the contig sequences to obtain the scaffold sequence; Gapfiller V2.1.1

(http://www.sourceforge.net/projects/gapfiller/) software was used to supplement gaps If gaps still exist, primers were designed, sequenced by PCR, reassembled until the complete pseudo genome sequence was obtained, and then the sequences were matched to the pseudo genome for genome correction. Finally, the corrected sequence was rearranged according to the cp structure to obtain the complete circular cp genome sequence.

\section{Structural annotation of the cp genome}

We used Blast v2.2.25 (http://blast.ncbi.nlm.nih.gov/Blast.cgi) software to compare the coding DNA sequence (CDS) of the cp genome in the NCBI database (http:// www.ncbi.nlm.nih.gov/genomes). The final cp genome gene annotation was obtained after manual correction. rRNA annotation information for cp genome was obtained using hmmer v3.1b2 (http://www.hmmer.org/) software to compare the rRNA sequence of cp genome in NCBI database. We used Aragorn v1.2.38 (http://130.235.244.92/aragorn/) software to predict the tRNA of the cp genome sequence and obtain the tRNA annotation information of the cp genome. OGDRAW (http://chlorobox.mpimp-golm.mpg.de/OGDraw.html) software was used to visualize the annotation results and draw a physical map of the cp genome. 


\section{IR/SC (Inverted repeat/Single-copy region) boundary comparison and codon preference analysis of cp genomes}

There are four boundaries between IR (Inverted repeat), LSC (Large single-copy region), and SSC (Small single-copy region). In the process of genome evolution, the IR boundary will expand and contract, pushing some genes into the IR region or a single copy region. We used Adobe Illustrator CS5 mapping software to map the annotation information of the cp genome of seven species of Nymphaea to the map of the simplified structure of the cp genome, which was used to compare the boundaries of four regions of the cp genome of Nymphaea, in order to show the extent of the shrinking and expanding of IR regions. The number of codons encoded by all protein coding genes and the relative synonymous codon usage (RSCU) in the cp genome of seven species of Nymphaea were calculated using Codonw software (http://codonw.sourceforge.net/). RSCU value $>1$ indicates that the codon is a preferred codon and is frequently used. RSCU $=1$ indicates that the codon has no preference. The value of RSCU $<1$ indicates that the frequency of using the codon is low.

\section{Repeat sequence and SSR Analysis}

We used Vmatch v2.3.0 (http://www.vmatch.de/) software to identify dispersed repeats. The SSR analysis, including single nucleotide SSRs, dinucleotide SSRs, trinucleotide SSRs, tetranucleotide SSRs, pentanucleotide SSRs, and hexanucleotide SSRs, was conducted using MISA V1.0 (MlcroSAtellite identification tool; http://pgrc.ipkgatersleben.de/misa/misa.html) software. The minimum repetition times of these SSRs were set to eight, five, three, three, three, and three, respectively.

\section{KaKs, nucleotide polymorphism, and global comparison analysis}

The gene sequence was compared using MAFFT V7.310 (https://mafft.cbrc.jp/alignment/software/) software, and the values of $\mathrm{Ka}$ and Ks were calculated by KaKs-Calculator V2.0 (https://sourceforge.net/projects/kakscalculator2/) software. Ka/Ks > 1 indicates positive selection; $\mathrm{Ka} / \mathrm{Ks}<1$ indicates purifying selection. The CDS sequences of the same gene in seven species of Nymphaea were compared globally using the MAFFT software, and the nucleotide polymorphism (Pi) of each gene was calculated using VCFtools. The cp genomes of seven species of Nymphaea were compared globally using MAFFT software, and then SNPs and indel were identified.

\section{Cp genome collinearity and phylogenetic tree analysis}

Using the cp genome of $N$. colorata as the reference sequence, the cp genomes of seven novel sequences from this study were compared using Mauve software to detect whether there was rearrangement in the cp genomes among the included Nymphaea species. Seven cp genes of the genus Nymphaea in this study and 14 Nymphaeaceae species published in NCBI database were selected for phylogenetic analysis. We carried out multiple sequence alignment using MAFFT software. The evolutionary tree was 
built using RAxMLv8.2.10 (https://cme.h-its.org/exelixis/software.html) software, and the GTR model and hill clipping algorithm were selected.

\section{Abbreviations}

Cp: chloroplast; SSR: simple sequence repeat; SNP: Single nucleotide polymorphism; CDS: Coding DNA sequence; IR: Inverted repeat; SC: Single-copy region; LSC: Large single-copy region; SSC: Small singlecopy region; RSCU: Relative synonymous codon usage; Pi: Nucleotide polymorphism; Ka: synonymous substitution rate; Ks: missense substitution rate; ITS: internal transcribed spacers.

\section{Declarations}

\section{Ethics approval and consent to participate}

Not applicable.

\section{Consent for publication}

Not applicable.

\section{Availability of data and materials}

The seven cp genomes sequences we obtained from this study were archived in NCBI. The accession numbers are MT107636, MT107632, MT107637, MT107633, MT107631, MT107634, and MT107635.

\section{Competing interests}

The authors declare that they have no competing interests.

\section{Funding}

This study was supported by the National Natural Science Foundation of China (31701948). The funder had no role in the design of the study and collection, analysis, and interpretation of data and in writing the manuscript.

\section{Authors' contributions}

CQS, FDC, NJT and ZLD designed the experiments. CQS and XS performed the experiments. CQS, NJT and ZLD analyzed the data. CQS wrote the manuscript. All authors have read and approved the final manuscript.

\section{Acknowledgements}

Not applicable 


\section{Author details}

1 Zhenjiang Institute of Agricultural Science in Jiangsu Hilly Areas, Jurong, 242400, China.

2 College of Horticulture, Nanjing Agricultural University, Nanjing 210095, China.

\section{References}

1. 1. Qiu YL, Lee J, Bernasconi-Quadroni F, Soltis DE, Chase M W. The earliest angiosperms: evidence from mitochondrial, plastid and nuclear genomes. Nature. 1999; 402(6760): 404-7.

2. Zanis MJ, Soltis DE, Soltis PS, Mathews S, Donoghue MJ. The root of the angiosperms revisited. Proceedings of the National Academy of Sciences of the United States of America. 2002; 99(10): 6848-53.

3. 3. Sun CQ, Ma ZH, Sun GS, Dai ZL, Teng NJ, Pan YP. Cellular mechanisms of reproductive barriers in some crosses of water lily (Nymphaea) cultivars. Hort Science. 2015; 50:30-5.

4. 4. Sun $C Q, M a Z H$, Zhang ZC, Sun GS, Dai ZL. Factors influencing cross barriers in interspecific hybridizations of water lily. Journal of the American Society for Horticultural Science. 2018; 143(2):1-6.

5. 5. Huang GZ, Deng HQ, Li Z, Li G. Water lily. Beijing: China Forestry Press; 2009.

6. 6. Löhne C, Borsch T, Wiersema JH. Phylogenetic analysis of Nymphaeales using fast-envolving and noncoding chloroplast Bot J Linn Soc. 2007; 154:141-63.

7. Borsch T, Hilu KW, Wiersema JH, Löhne C, Barthlott W, Wilde V. Phylogeny of Nymphaea (Nymphaeaceae): evidence from substitutions and microstructural changes in the chloroplast trnTtrnF region. Int J Plant Sci. 2007; 168:639-71.

8. Dkhar J, Kumaria S, Rao SR, Tandon P. Sequence characteristics and phylogenetic implications of the nrDNA internal transcribed spacers (ITS) in the genus Nymphaea with focus on some indian representatives. Plant Systematics and Evolution. 2012; 298(1): 93-108.

9. Guisinger MM, Kuehl JV, Boore JL, Jansen RK. Extreme reconfiguration of plastid genomes in the angiosperm family Geraniaceae: rearrangements, repeats, and codon usage. Molecular biology and evolution. 2011; 28(1):583-600.

10. Blazier JC, Ruhlman TA, Weng ML, Rehman SK, Sabir JSM, Jansen RK. Divergence of RNA polymerase a subunits in angiosperm plastid genomes is mediated by genomic rearrangement. Sci Rep. 2016; 6: 24595.

11. Dong WP, Xu C, Cheng T, Lin K, Zhou SL. Sequencing angiosperm plastid genomes made easy: a complete set of universal primers and a case study on the phylogeny of Saxifragales. Genome Biol Evol. 2013; 5: 989-97.

12. Lee SR, Kim K, Lee BY, Lim CE. Complete chloroplast genomes of all six Hosta species occurring in Korea: molecular structures, comparative, and phylogenetic analyses. BMC Genomics. 2019; 20:833. 
13. Duan H, Guo JB, Xuan L, Wang ZY, Li MZ, Yin YL, Yang Y. Comparative chloroplast genomics of the genus Taxodium. BMC Genomics. 2020; 21:114.

14. Terakami S, Matsumura Y, Kurita K, Kanamori H, Katayose $Y$, Yamamoto T, Katayama H. Complete sequence of the chloroplast genome from pear (Pyrus pyrifolia): genome structure and comparative analysis. Tree Genet Genom. 2012; 8:841-54.

15. Jansen RK, Christopher S, Seung-Bum L, Hansen AK, Henry D. Complete plastid genome sequences of three rosids (Castanea, Prunus, Theobroma): evidence for at least two independent transfers of rp/22 to the nucleus. Molecular Biology and Evolution. 2011; 28(1): 835-47.

16. Guisinger MM, Chumiley TW, Kuehl JV, Boore JL, Jansen RK. Implications of the plastid genome sequence of Typha (Typhaceae, Poales) for understanding genome evolution in Poaceae. Journal of Molecular Evolution. 2010; 70(2):149-66.

17. Gichira AW, Li ZZ, Saina JK. The complete chloroplast genome sequence of an endemic monotypic genus Hagenia (Rosaceae): structural comparative analysis, gene content and microsatellite detection. Peer j. 2017; 5(6):e2846.

18. Liu LX, Li R, Worth JRP, Xian L, Pan L, Gameron KM, Fu CX. The complete chloroplast genome of Chinese Bayberry (Morella rubra, Myricaceae): implications for understanding the evolution of Fagales. Frontiers in Plant Science. 2017; 8:968.

19. Kim KJ, Lee HL. Complete chloroplast genome sequences from Korean ginseng (Panax schinseng Nees) and comparative analysis of sequence evolution among 17 vascular plants. DNA Research. 2004; 11(4):247-61.

20. Lin CP, Wu CS, Huang YY, Chaw SM. The complete chloroplast genome of Ginkgo biloba reveals the mechanism of inverted repeat contraction. Genome biology and evolution. 2012; 4(3):374-81.

21. Lu RS, Li P, Qiu YX. The complete chloroplast genomes of three Cardiocrinum (Liliaceae) species: comparative genomic and phylogenetic analyses. Frontiers in Plant Science. 2017; 7:2054.

22. Li P, Lu RS, Xu WQ, Ohi-Toma T, Cai MQ, Qiu YX, Cameron KM, Fu CX. Comparative genomics and phylogenomics of east Asian Tulips (Amana, Liliaceae). Frontiers in Plant Science. 2017; 8: 451.

23. Shimada $H$, Sugiura M. Fine structural features of the chloroplast genome: comparison of the sequenced chloroplast genomes. Nucleic Acids Res. 1991; 19: 983-95.

24. Zhou M, Long W, Li X. Patterns of synonymous codon usage bias in chloroplast genomes of plants. Forest Ecosystems. 2008; 4:235-42.

25. Ren T, Yang YC, Zhou T, Liu ZL. Comparative plastid genomes of Primula species: sequence divergence and phylogenetic relationships. International Journal of Molecular Sciences. 2018; 19(4):1050.

26. Morton BR. Selection on the codon bias of chloroplast and cyanelle genes in different plant and algal lineages. Journal of Molecular Evolution. 1998; 46(4):449-59.

27. Yang Y, Zhou T, Duan D, Feng L, Zhao G. Comparative analysis of the complete chloroplast genomes of five Quercus species. Frontiers in plant science. 2016; 7: 959. 
28. Hu Y, Woeste KE, Zhao P. Completion of the chloroplast genomes of five Chinese Juglans and their contribution to chloroplast phylogeny. Frontiers in Plant Science. 2016; 7: 1955.

29. Weng ML, Blazier JC, Govindu M, Jansen RK. Reconstruction of the ancestral plastid genome in Geraniaceae reveals a correlation between genome rearrangements, repeats, and nucleotide substitution rates. Molecular Biology and Evolution. 2013; 31(3):645-59.

30. Kuang DY, Wu H, Wang YL, Gao LM, Zhang SZ, Lu L. Complete chloroplast genome sequence of Magnolia kwangsiensis (Magnoliaceae): implication for DNA barcoding and population genetics. Genome. 2011; 54: 663-73.

31. Martin G, Baurens FC, Cardi C, Aury JM, D'Hont A. The complete chloroplast genome of banana (Musa acuminata, Zingiberales): insight into plastid monocotyledon evolution. PloS One. 2013; 8:e67350.

32. Shaw J, Shafer HL, Leonard OR, Kovach MJ, Schorr M, Morris AB. Chloroplast DNA sequence utility for the lowest phylogenetic and phylogeographic inferences in angiosperms: the tortoise and the hare IV. American journal of Botany. 2014; 101(11):1987-2004.

33. Niu Z T, Xue QY, Zhu SY, Sun JJ, Liu W, Ding XY. The complete plastome sequences of four orchid species: insights into the evolution of the orchidaceae and the utility of plastomic mutational hotspots. Frontiers in plant science. 2017; 8:715.

34. Wolfe KH. Similarity between putative ATP-binding sites in land plant plastid ORF2280 proteins and the FtsH/CDC48 family of ATPases. Current Genetics. 1994; 25(4):379-83.

35. Drescher A, Ruf S, Calsa TJ, Carrer H, Bock R. The two largest chloroplast genome-encoded open reading frames of higher plants are essential genes. The Plant Joural. 2000; 22(2):97-104.

36. Wang B, Gao L, Su YJ, Wang T. Adaptive evolutionary analysis of chloroplast genes in euphyllophytes based on complete chloroplast genome sequences. Acta Scientiarum Naturalium Universitatis Sunyatseni. 2012; 51(3):108-13.

37. Les D H, Schneider E L, Padgett $D$ J. Phylogeny, classification and floral evolution of water lilies (Nymphaeales): A synthesis of non-molecular, rbcL, matK and 18S rDNA data. Systematic Botany. 1999; 24:28-46.

38. Tamura M. Relationship of Barclaya and classification of Nymphaeales. Acta phytotaxonomica et geobotanica. 1982; 33:336-45.

39. Moseley MF, Schneider EL, Williamson PS. Phylogenetic interpretations from selected floral vasculature characters in the Nymphaeaceae sensu lato. Aquatic Botany. 1993; 44:325-42.

40. Songpanic P, Hongtrakul V. Intersubgeneric cross in Nymphaea spp. L. to develop a blue hardy waterlily. Scientia Horticulturae. 2010; 124:475-81.

41. Li SJ, Yu Q, Chen C, Zhang Y, Wu YP, Yu G. Breeding progress of waterlilies in China. Journal of plant genetic resources. 2019; 20(4): 829-35.

42. Sun CQ, Chen FD, Teng NJ, Yao YM, Shan X, Dai ZL. Transcriptomic and proteomic analysis reveals mechanisms of low pollen-pistil compatibility during water lily cross breeding. BMC Plant Biology. 2019; 19:542. 


\section{Tables}

Table 1 Summary statistics for the assembly of seven Nymphaea species chloroplast genomes

\begin{tabular}{|c|c|c|c|c|c|c|c|}
\hline $\begin{array}{l}\text { Genome features } \\
\text { Voucher ID }\end{array}$ & $\begin{array}{l}\text { N. odorata } \\
\text { ZJWL-031 }\end{array}$ & $\begin{array}{l}\text { N.tetragona } \\
\text { ZJWL-036 }\end{array}$ & $\begin{array}{l}\text { N. rubra } \\
\text { ZJWL-008 }\end{array}$ & $\begin{array}{l}\text { N. gigantea } \\
\text { ZJWL-014 }\end{array}$ & $\begin{array}{l}\text { N. potamophila } \\
\text { ZJWL-021 }\end{array}$ & $\begin{array}{l}\text { N. colorata } \\
\text { ZJWL-041 }\end{array}$ & $\begin{array}{l}\text { N. micrantha } \\
\text { ZJWL-043 }\end{array}$ \\
\hline NCBI accession NO. & MT107636 & MT107634 & MT107632 & MT107637 & MT107633 & MT107631 & MT107635 \\
\hline Genome size $₫ b p \square$ & 159968 & 159956 & 159322 & 160179 & 159232 & 159842 & 159900 \\
\hline LSC size[bp] & 90025 & 89837 & 89564 & 90266 & 89450 & 89969 & 89950 \\
\hline SSC size $\llbracket b p \square$ & 19533 & 19655 & 19340 & 19541 & 19456 & 19547 & 19576 \\
\hline IR size $[\mathrm{bp} \square$ & 25205 & 25232 & 25209 & 25186 & 25163 & 25163 & 25187 \\
\hline Number of genes & 126 & 128 & 127 & 129 & 129 & 129 & 129 \\
\hline Protein genes [unique] & $81(75)$ & 83ロ77ロ & $82(76)$ & 84(78) & $84(78)$ & 84(78) & 84(78) \\
\hline tRNA genes [unique] & $37(30)$ & $37 \square 30 \square$ & $37(30)$ & $37(30)$ & $37(30)$ & $37(30)$ & $37(30)$ \\
\hline rRNA genes [unique] & $8(4)$ & $8 \llbracket 4 \square$ & $8(4)$ & $8(4)$ & $8(4)$ & $8(4)$ & $8(4)$ \\
\hline Duplicated genes in IR & 32 & 32 & 32 & 32 & 32 & 32 & 32 \\
\hline GC content (\%) & 39.08 & 39.14 & 39.09 & 39.11 & 39.13 & 39.14 & 39.16 \\
\hline GC content in LSC $(\%)$ & 37.76 & 37.82 & 37.73 & 37.70 & 37.79 & 37.77 & 37.80 \\
\hline GC content in SSC $(\%)$ & 34.19 & 34.31 & 34.20 & 34.44 & 34.28 & 34.42 & 34.38 \\
\hline GC content in IR $(\%)$ & 43.35 & 43.37 & 43.37 & 43.44 & 43.39 & 43.43 & 43.44 \\
\hline
\end{tabular}

Table 2 List of genes in $N$. gigantea, $N$. potamophila $N$. colorata and $N$. micrantha chloroplast genomes. 


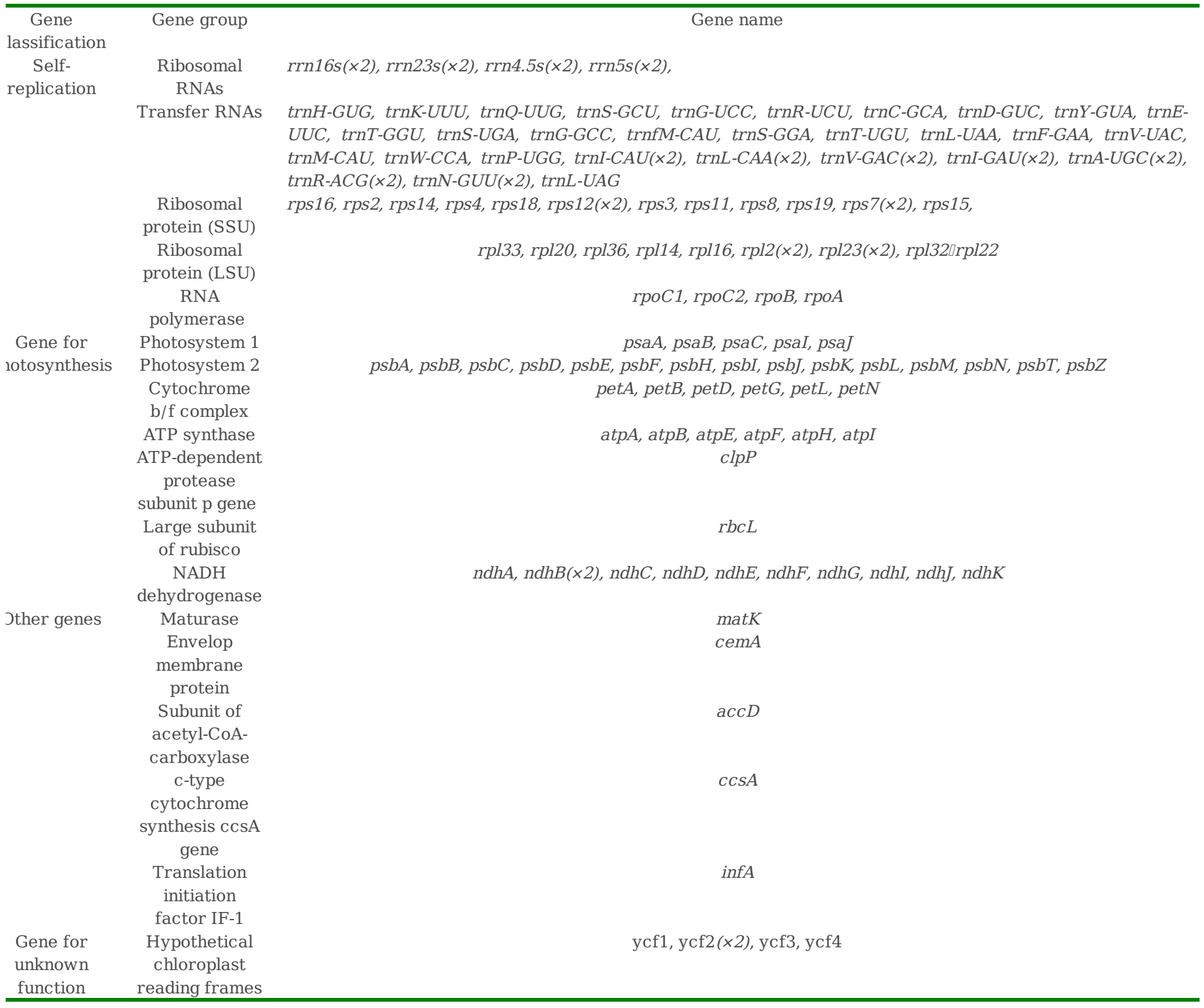

Table 3 Summary of dispersed repeats and SSRs in seven Nymphaea species 


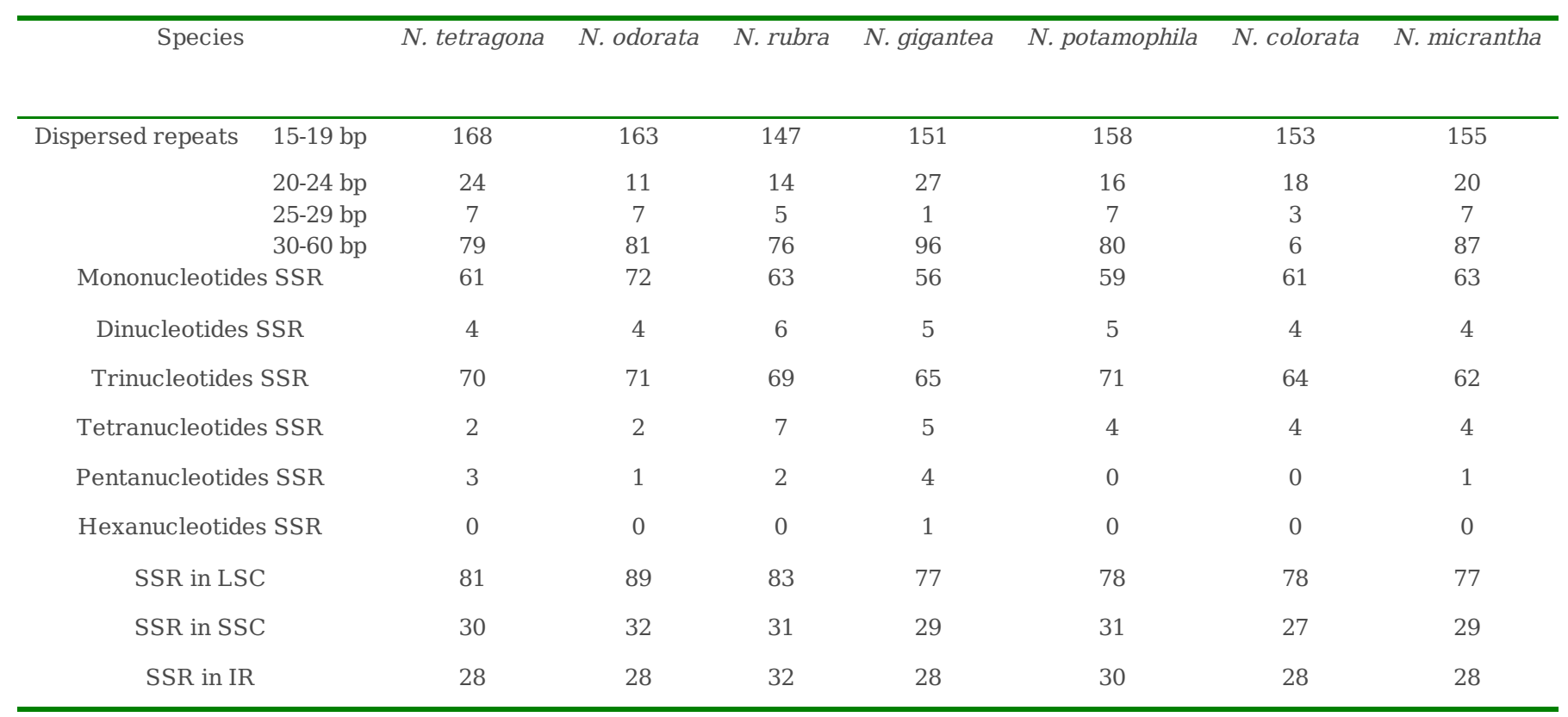

Figures 


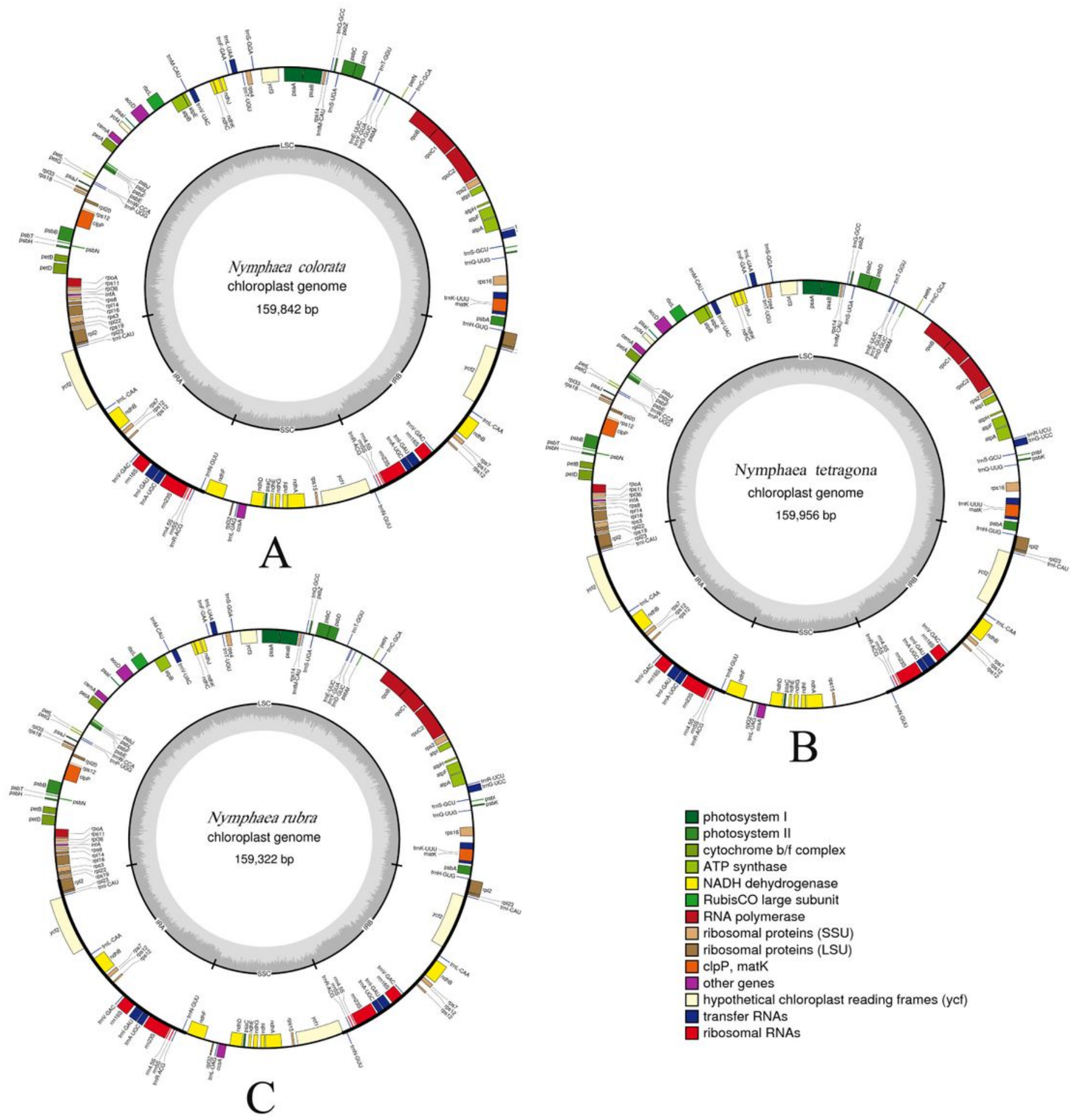

Figure 1

Seven novel cp genome sequences had the classical quadripartite structure that contained one LSC, one $\mathrm{SSC}$, and two IR (IRa and IRb) regions 


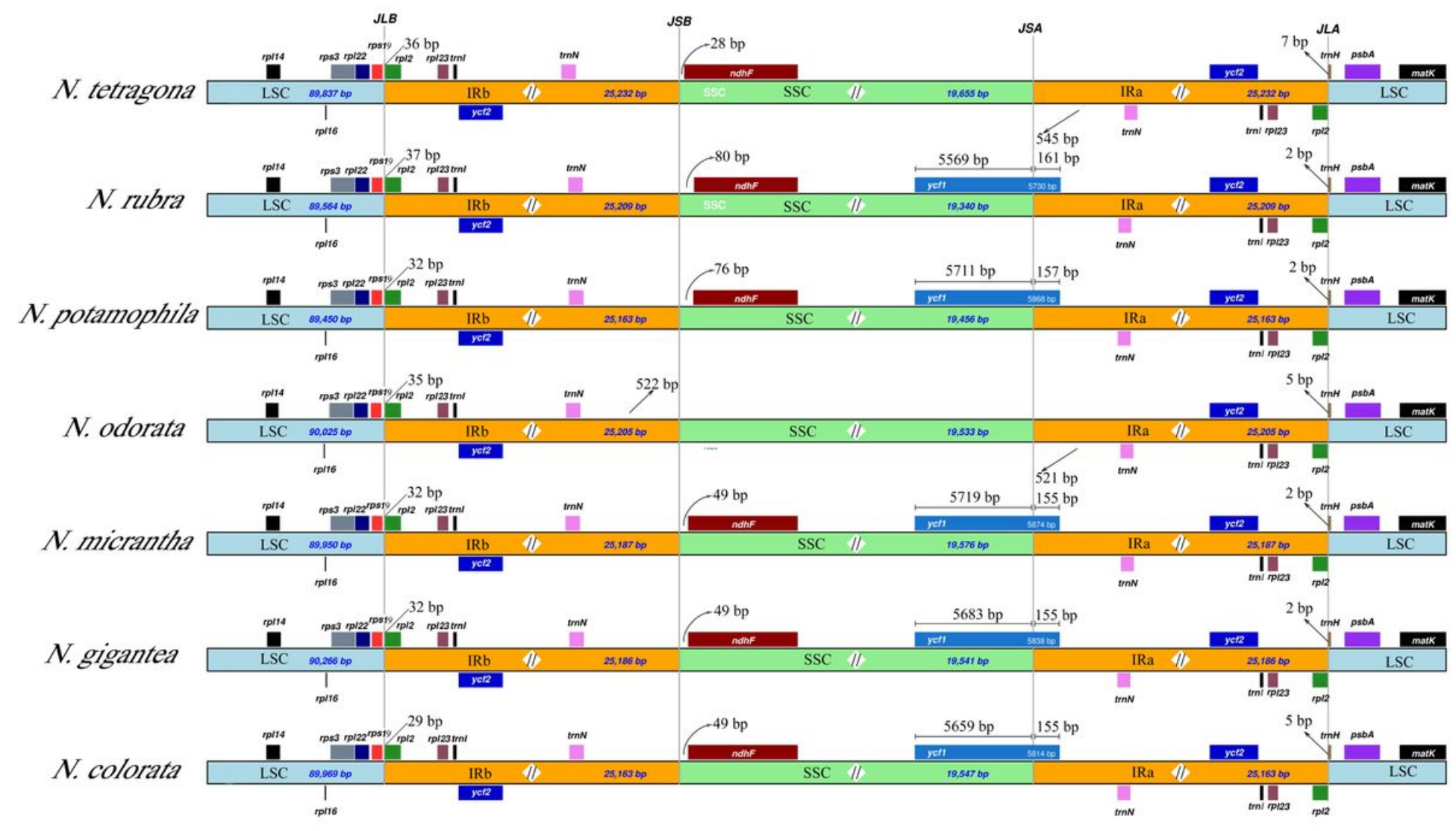

Figure 2

The LSC/IRb boundaries of the seven Nymphaea species were all within the rpl2 gene, and the length of the overlapping region between the boundary and the rpl2 gene was 15-39 bp.

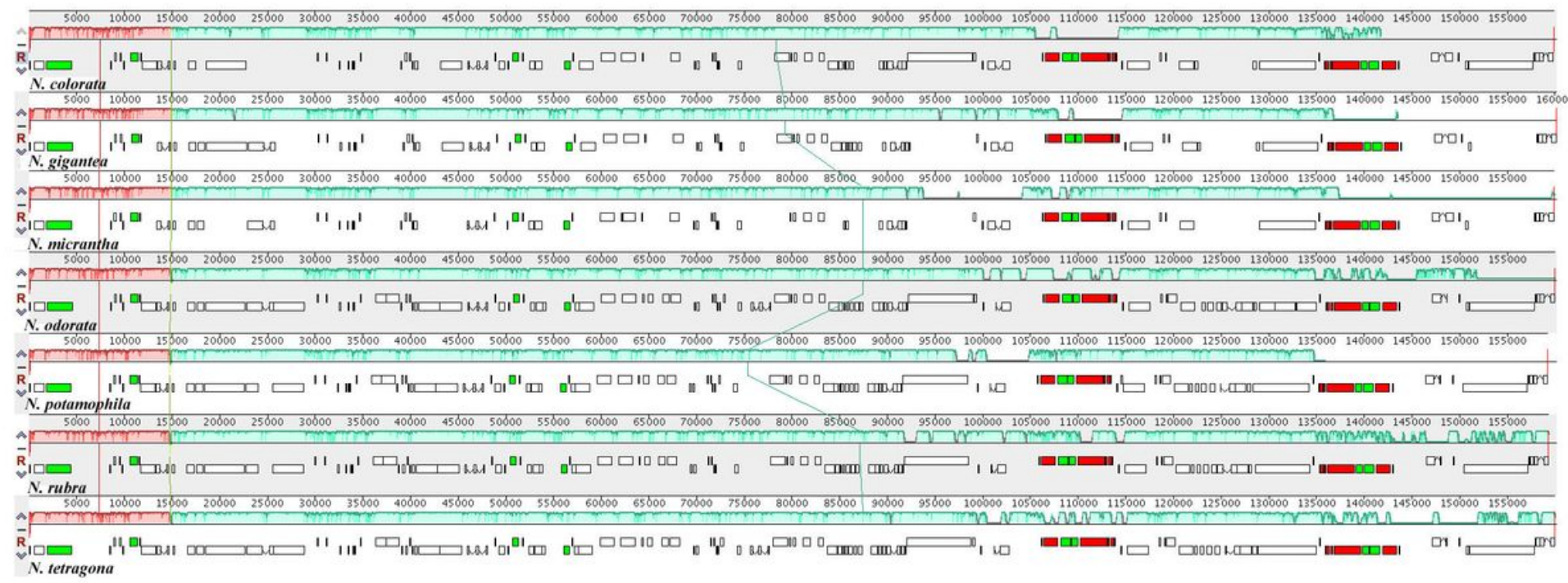

\section{Figure 3}

The sequence differences of 105-115 kb of the cp genome of the seven species of Nymphaea 


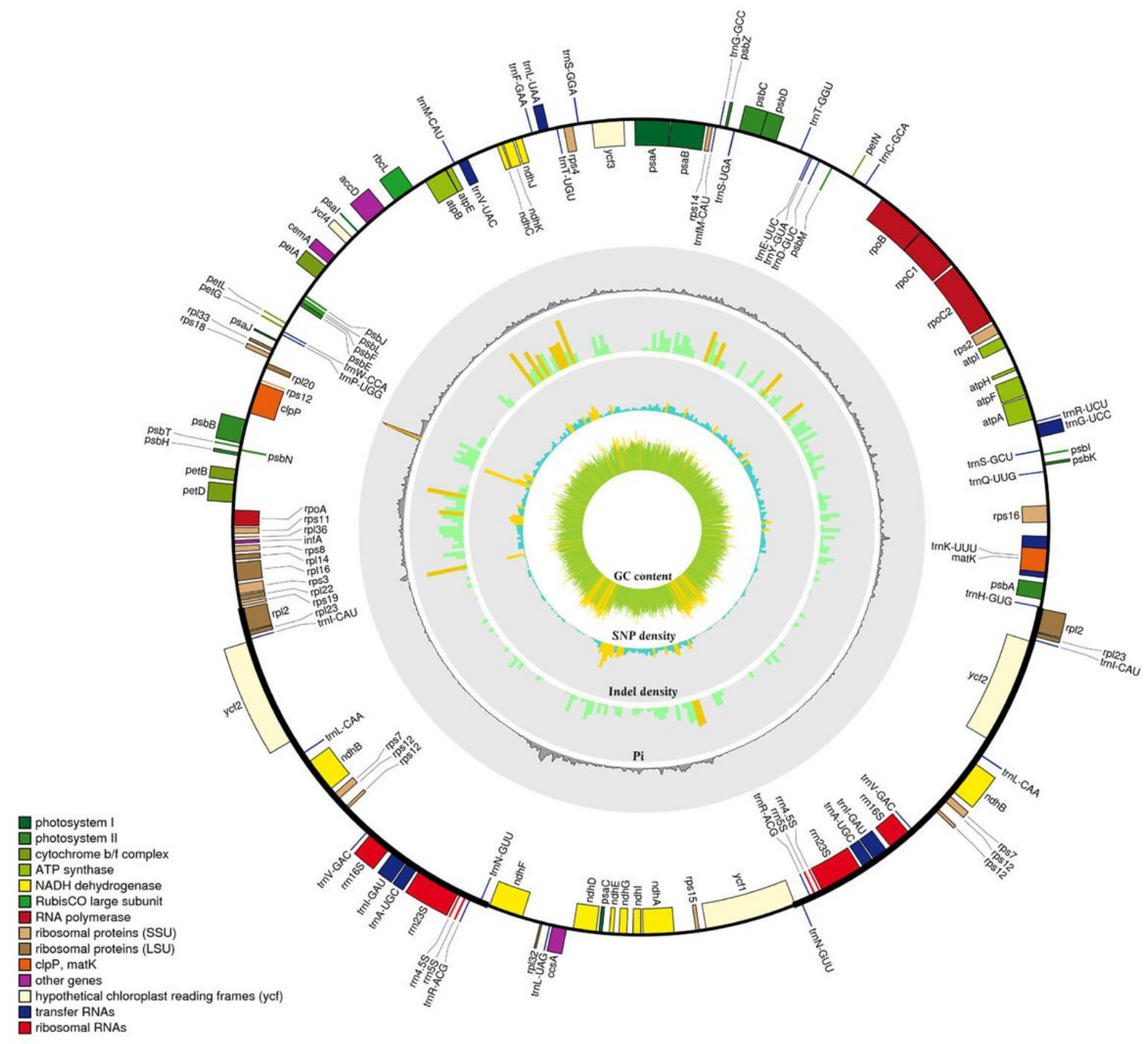

Figure 4

A relatively large number of SNPs in some highly variable regions located in rpoA-rpl20, rbcL-ndhC, ndhD-ndhF, and trnN-GUU-ndhA 


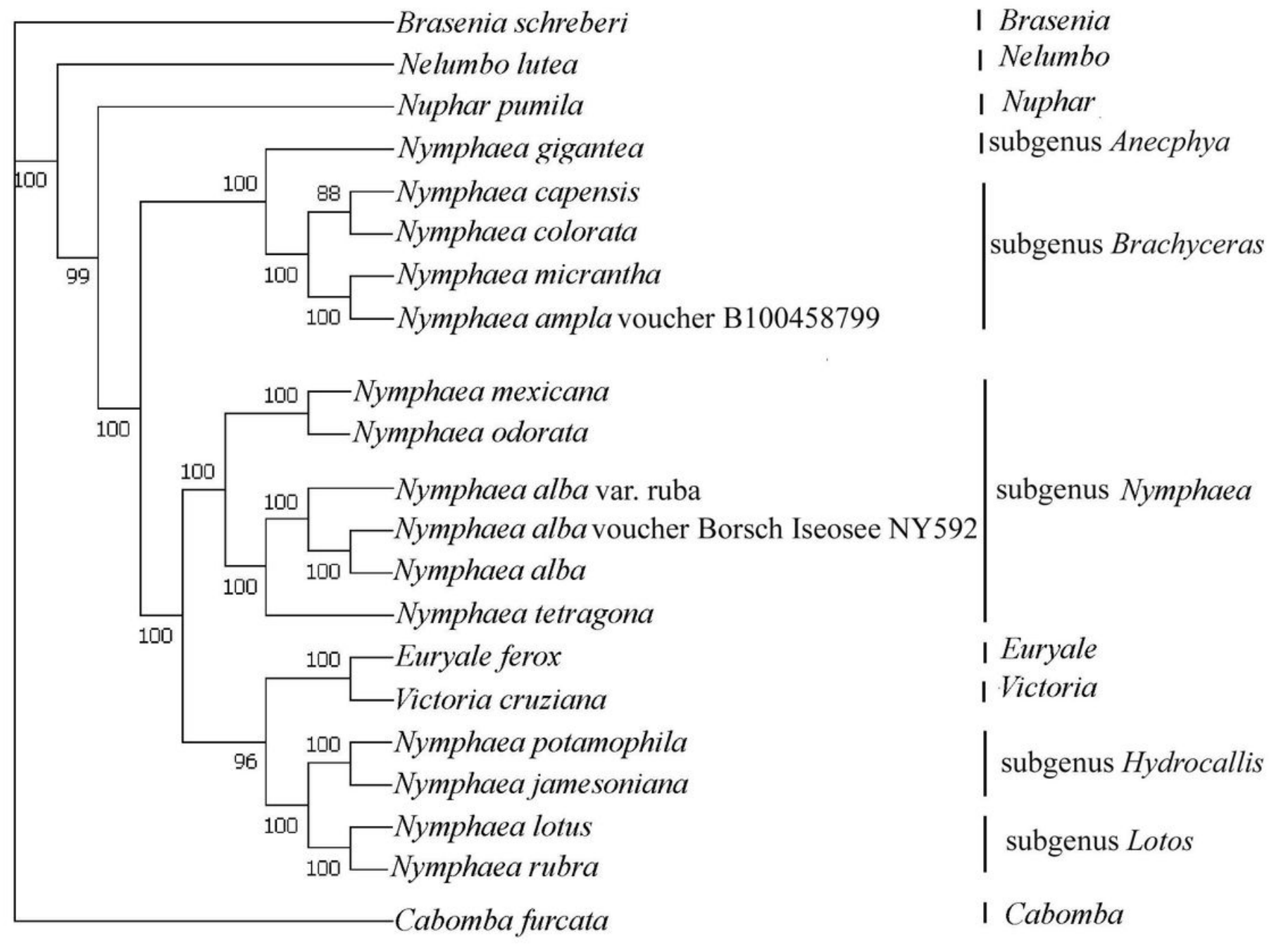

Figure 5

Five Nymphaea subgenera

\section{Supplementary Files}

This is a list of supplementary files associated with this preprint. Click to download.

- Additionalfile1.xls

- Additionalfile2.xls

- Additionalfile5.xIs

- Additionalfile4.xlt

- Additionalfile3.xls 